\title{
A computational framework to explore cellular response mechanisms from multi-omics datasets
}

James C. Pino ${ }^{1, \dagger}$, Alexander L. R. Lubbock ${ }^{2, \dagger}$, Leonard A. Harris ${ }^{2}$, Danielle B. Gutierrez ${ }^{2}$,

Melissa A. Farrow ${ }^{3}$, Nicole Muszynski ${ }^{4,6}$, Tina Tsui ${ }^{2}$, Jeremy L. Norris ${ }^{2,5}$, Richard M.

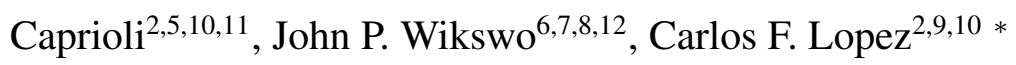

${ }^{1}$ Chemical and Physical Biology Graduate Program, Vanderbilt University, Nashville, TN, USA

${ }^{2}$ Department of Biochemistry, Vanderbilt University School of Medicine, Nashville, TN, USA

${ }^{3}$ Department of Pathology, Microbiology, and Immunology, Vanderbilt University School of Medicine, Nashville, TN, USA

${ }^{4}$ Department of Surgery, Vanderbilt University School of Medicine, Nashville, TN, USA

${ }^{5}$ Department of Chemistry, Vanderbilt University, Nashville, TN, USA

${ }^{6}$ Department of Physics and Astronomy, Vanderbilt University, Nashville, TN, USA

${ }^{7}$ Department of Molecular Physiology and Biophysics, Vanderbilt University, Nashville, TN, USA

${ }^{8}$ Department of Biomedical Engineering, Vanderbilt University, Nashville, TN, USA

${ }^{9}$ Department of Biomedical Informatics, Vanderbilt University School of Medicine, Nashville, TN,

\section{USA}

${ }^{10}$ Department of Pharmacology, Vanderbilt University School of Medicine, Nashville, TN, USA

${ }^{11}$ Department of Medicine, Vanderbilt University, Nashville, TN, USA

${ }^{12}$ Vanderbilt Institute for Integrative Biosystems Research and Education, Vanderbilt University, Nashville, TN, USA

\footnotetext{
*To whom correspondence should be addressed: Tel: +1 (615) 936-1311; Email: c.lopez@ vanderbilt.edu
} 
bioRxiv preprint doi: https://doi.org/10.1101/2020.03.02.974121; this version posted March 3, 2020. The copyright holder for this preprint (which was not certified by peer review) is the author/funder, who has granted bioRxiv a license to display the preprint in perpetuity. It is made available under aCC-BY-NC 4.0 International license.

\section{$\dagger$ These authors contributed equally to this work.}


Recent technological advances have made it feasible to collect multi-condition transcriptome and proteome time-courses of cellular response to perturbation. The increasing size and complexity of these datasets impedes mechanism of action discovery due to challenges in data management, analysis, visualization, and interpretation. Here, we introduce MAGINE, a software framework to explore complex time-course multi-omics datasets and build mechanistic hypotheses of dynamic cellular response. MAGINE combines data management, enrichment, and network analysis and visualization within an interactive, Jupyter notebookbased environment to enable human-in-the-loop inquiry of complex datasets. We demonstrate how measurements from HL-60 cellular response to bendamustine treatment can be used to build a mechanistic, multi-resolution description of cellular commitment to fate. We present a systems-level description of signal execution from cellular DNA-damage response, to cell cycle arrest, and eventual commitment to apoptosis, mediated by over 2000 biochemical species. We further show that MAGINE can reveal unexpected, non-canonical effects of bendamustine treatment, including disruption of cellular pathways relevant to HIV infection response. MAGINE is available from https://github.com/lolab-vu/magine.

\section{Introduction}

Cellular response to perturbations can elicit molecular responses across multiple processes such as gene expression modulation, changes in protein and metabolic activity, and in extreme cases, 
changes in DNA structure (e.g., mutations). Modern, accessible technologies—most notably mass spectrometry (MS) and RNA-sequencing (RNAseq)— have enabled the measurement of biochemical interactions at molecular resolution for whole cellular genomes, proteomes, and metabolomes 1+3. Recent work by our labs and others have already shown the potential of these kinds of datasets to gain a systems-level understanding of cellular response mechanisms to perturbations, with measurements that can easily number in the thousands to millions of data points $4-6$. Although these measurements in principle contain the molecular details necessary to formulate mechanistic hypotheses about cellular response to perturbations, the analysis of these datasets currently entails multiple tools, most notably enrichment- and network-based methods.

Enrichment analysis can provide insights about relevant cellular processes by comparing multiple experimental conditions following perturbations such as drug treatments 78 . This approach can be used to identify biological processes with altered activity by identifying groups of genes or proteins that are up- or down-regulated following treatment. Unfortunately, for the purposes of mechanistic exploration, these approaches fail to provide insights about molecular interactions that could drive a specific cellular process. For the purposes of large multi-omics and multi-experiment exploration, popular web-based enrichment analysis tools such as EnrichR ${ }^{9}$ and Webgestalt 10 can only handle one sample at a time through their web interfaces, thus posing a major limitation given the high-throughput needed for multi-omics analysis.

In contrast to enrichment-based analysis methods, network-based analysis produces "maps" of biochemical species and their interactions. Networks can then be explicitly analyzed - e.g. 
using graph theoretic methods - to find paths or groups of relevant interactions between two or more network points. However, these become difficult to visualize and interrogate when the graph grows beyond a few tens of nodes, as seen in genome-wide networks. leading to the familiar "hairball" problem. Network tools, most notably Cytoscape 11, partially address the needs for combined enrichment and network analysis through the use of plugins, but their capabilities for multi-sample analysis are limited. Ingenuity Pathway Analysis 12 , a useful pathway analysis tool available for multi-omics data, can provide cellular process exploration, but its closed, proprietary nature limits extension by users to meet the needs of the field.

Recognizing the need for multi-omics analysis, other tools have been built that can handle small multi-omics datasets $910[13,15$. However, these tools were not designed for the emerging needs posed by large and complex, multi-time point or multi-sample datasets. Thus there is an unmet need for novel tools to $(i)$ integrate -omics datasets from multiple experimental modalities, (ii) provide a platform where multiple analysis tools can be used in tandem, and (iii) enable humanguided mechanism exploration within a reproducible, shareable workflow environment $16-21$

To address the needs for modern multi-omics data analysis, we developed the Mechanism of

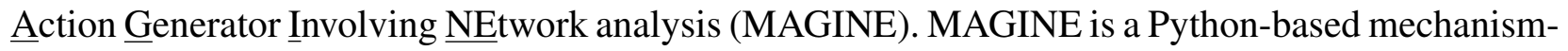
of-action exploration framework that unifies enrichment and network analyses, enabling the user to explore interactions across multiple cellular processes along with the molecular interactions that drive these processes. MAGINE embraces a literate programming paradigm ${ }^{22}$ in which biological knoweldge, data exploration, and visualization are integrated within a live document within an ex- 
ecutable web-based environment such as Jupyter notebooks, thus facilitating access to the Python scientific ecosystem.

To demonstrate the capabilities of MAGINE, we explore the time-course response of HL-60 cells to bendamustine treatment. Bendamustine is a well-established DNA-damage agent used for cancer treatments in the clinic with a consensus mechanism of action ${ }^{23}$. Our analysis reveals detailed, systems-level, dynamic molecular mechanisms, which comprise thousands of biochemical interactions across multiple cellular processes. These results significantly expand upon the consensus mechanism accepted for Bendamustine in cellular perturbations, which comprises a few tens of molecular interactions 23,24 . We also demonstrate how MAGINE can be used to explore bendamustine side-effects; specifically, protein interactions that could provide a mechanistic explanation for previous reports of bendamustine treatments in HIV patients. Finally, all our MAGINE-based analysis is documented using Jupyter notebooks, which offer a means to transparently report complete analyses, suitable for distribution across members of the scientific community to evaluate and expand on as desired. In summary, MAGINE unifies multiple practices in the field onto a common, open, scalable platform that enables the extraction of cellular response mechanisms from large, complex -omics datasets.

\section{Results}

MAGINE: A framework to explore cellular response mechanisms using multi-omics MAGINE is implemented in Python, a suitable language for a biological exploration platform due to its 
ease of use, large user base, and integration with over 200,000 packages in the Python Package Index as of the writing of this manuscript. The platform has been tested on Windows, Mac OS, and Linux. It can be used within the Python console, interactive Jupyter Notebooks, or within data processing and analysis pipelines. We envisage the majority of users are best served through the Jupyter notebook option, and thus describe that in the most detail, including a tutorial notebook (Supplementary Information).

MAGINE comprises three main modules (Figure 1): data management and visualization, enrichment analysis, and network analysis. Each module can be used independently, but data can be easily shared across modules. We present a brief summary of each module below, followed by an applied case study.

Data analysis module The data management module handles data storage, access, and analysis. MAGINE utilizes the $\operatorname{pandas}^{25}$ library to provide database-like capabilities for -omics data querying. Data are loaded using a tabular, comma-separated values (CSV) file, with one measurement per row (see Methods). MAGINE stores these in an ExperimentalData class, which provides a simple, high-level interface to access, filter, and search these data as needed. This module also provides visualization capabilities, which include sample comparisons, time-series clustering, and species differential expression trends over time (Figure 1). A summary of these methods is shown in Supplementary Table $\mathbf{S 2}$, and example usage can be found online or in Supplementary Notebook S1. 
Enrichment module The enrichment analysis module identifies over- or under-represented biochemical species that share common annotation (e.g., biological processes or molecular functions)

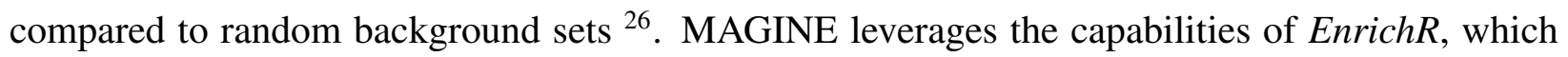
includes over 120 gene set "libraries"927. For analysis, users provide one or more lists of genes, which can be manually constructed or created by the ExperimentalData class (e.g., all up-regulated genes, species detected on a specific experimental platform, or filtered by time point). MAGINE automates analysis through EnrichR, as shown in Supplementary Figure S2. A single command is provided to query EnrichR across all time points and data platforms present in a dataset. The results are stored in an EnrichmentResult class, which includes methods to further query, filter, and visualize enrichment terms. Terms can be compared, ranked, grouped, and visualized with built-in methods (e.g., time-series heat map, word cloud). Genes corresponding to each term can be extracted and used to subset ExperimentalData or create subgraphs.

Even through the use of multiple databases, traditional enrichment analyses can yield terms of varied granularity, ranging from very broad (e.g., "biological process"), to highly specific (e.g., “cysteine-type endopeptidase inhibitor activity involved in apoptotic process"), within a single results output. The problem is exacerbated due to each gene mapping to multiple terms, thus introducing term redundancy, increasing the total number of explorable terms and hindering human interpretation. To address these issues, we developed an ontology compression method to aggregate terms based on gene content similarity (described in Methods). This significantly reduces enrichment term redundancy, which in turn greatly aids human interpretation. For example, on the bendamustine dataset analyzed herein, 84 terms from traditional enrichment analysis of our data 
can be compressed to 17 terms, an $80 \%$ reduction (see Results). A summary of functions available in the enrichment module is shown in Supplementary Table $\mathbf{S 3}$, and example usage can be found online or in Supplementary Notebook S3].

Network module MAGINE's network module allows users to build, query, and visualize molecular and gene annotation networks. A summary of the network module's methods is shown in Supplementary Table $\mathbf{S 4}$ and example usage can be found online or in Supplementary Notebook S2. The network module utilizes connectivity information from multiple databases, including

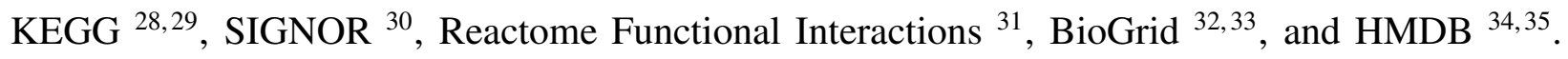
The graphs underlying these database are merged into a single network, which can be used to perform queries (e.g., find paths between nodes, apply clustering methods) or construct contextspecific networks based on a user-provided seed species list. Seed nodes can be obtained from various sources: significantly changed species, mutational evidence, literature review, or manual curation. The module iterates through the databases and expands the network by adding edges and nodes based on connectivity to the seed species in those databases (Supplementary Figure S4). The resulting networks are a subset of the background network, focused around the specific molecular seed species. These networks can be large ( $>20000$ nodes and $>100000$ edges). MAGINE users can use the Subgraph class to generate subnetworks (Supplementary Table S4). For example, these functions enable users to find paths between species, or find neighbors (upstream or downstream) of nodes of interest. Options are provided to limit the network expansion, such as setting a maximum distance from specific nodes. 
Additionally, we introduce a method to create a coarse-grained network from gene sets, which we refer to as an Annotated Gene-set Network (AGN). The AGN is motivated by the desire to combine dynamic, high-level information about biological processes from enrichment analysis with inter-process communication provided by molecular networks. This results in a coarsegrained network, where nodes are biological process terms and the edges are connections between the sets of nodes in the molecular network. This can be expanded into a fine-grained network, which contains the chemical species and their connections, thus enabling multi-resolution exploration. MAGINE's network module also provides various tools for network visualization, allowing users to overlay data or update network attributes. Users can visualize networks in Jupyter note-

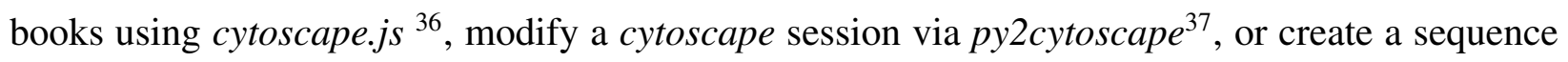
of figures of network activity through matplotib ${ }^{38}$, igraph ${ }^{39}$, or graphviz ${ }^{40}$. Networks can be exported using network ${ }^{41}$, for further external manipulation.

\section{Case study: Using MAGINE to explore the temporal response of bendamustine-treated leukemia}

cells. To demonstrate the capabilities of MAGINE, we extracted the mechanism of action from a multi-omics dataset containing molecular measurements for the response of HL-60 cells $\stackrel{42}{4}$ to treatment with bendamustine, a nitrogen mustard alkylating compound used for the treatment of chronic lymphocytic leukemia and non-Hodgkin's lymphoma ${ }^{24}$. Bendamustine is a cytotoxic agent that can induce DNA interstrand cross links (ICLs), which inhibits replication and transcription, leading to cell cycle arrest, and ultimately, cell death 2324 .

The main goal of this study was to probe and expand the consensus understanding of molecu- 
lar species and biological processes involved in cellular response to bendamustine treatment. Data were gathered across three proteomics platforms (label-free, SILAC, phospho-SILAC/phSILAC) and RNAseq, spanning between 30 seconds and 72 hours post-drug exposure with matching untreated controls (see Methods; Supplementary Figure S1). The analysis is summarized and documented in Supplementary Notebook $\mathrm{S} 1$.

In total, we detected 54818 unique molecular species across all platforms, of which 14426 were significantly changed versus control in at least one time point upon drug treatment. By platform, we saw 5115 changed species versus control from phSILAC MS, 1653 from label-free MS, 133 from SILAC MS, and 736 from RNAseq (Supplementary Figure $\mathbf{S 1}$ ). Early changes were detected at the phosphorylation level; phSILAC detected $>700$ such changes at the 30 s time point. Phosphorylation events occurred relatively evenly across all sample time points, while a gradual increase of significant differences versus control occurred over time in overall protein and RNA (Supplementary File S1). We next examined the overlap between significantly changed species across the experimental platforms (Supplementary Figure $\mathbf{S 1}$ c and d). The highest overlap among proteomic platforms was between phSILAC and label-free (643 species). Only 88 species were significantly changed versus respective controls in both RNA and at least one of the proteomic platforms (phSILAC, SILAC, and label-free; Supplementary Figure S1). Indeed, even within the protein platforms, a majority ( $>2500$ ) of measured species were unique to a single platform, while 688 species were measured in at least two platforms, and only 22 species were measured in all three platforms. Within-platform detection is generally reliable and repeatable ${ }^{4}$, thus the low overlap between significant species changes across platforms demonstrates the value of integrative, multi- 
platform analysis.

We proceeded to further analyze the dataset with a custom MAGINE workflow, integrating both network and enrichment analyses (Figure 2). First, we constructed a master signaling network utilizing significantly changed species across all platforms and time points as seed species. Separately, we performed an enrichment analysis across 52 reference gene sets (provided by EnrichR) for each time point and experimental platform; MAGINE automates this entire process using a single instruction, run_enrichment (). Each enrichment result set was arranged by three change types: up-regulated, down-regulated, and significantly changed (both up- and downregulated) species; and across 12 time points and four experimental platforms, resulting in 78 total result sets (not all combinations are represented). In total, there were 20758 enriched terms across the 52 reference gene sets with an adjusted p-value $\leq 0.05$.

We initially focused on phospho-protein changes to elucidate signaling activity. Using the MAGINE filtering capability, we selected only enriched terms originating from phSILAC data with the Reactome 2016 reference gene set, resulting in 561 unique terms. Next, we required terms to be significantly enriched in at least 3 of 5 time points, resulting in 84 terms. To reduce redundancy of closely-related terms (e.g., "cell cycle”, "cell cycle, mitotic”), we applied the remove_redundancy() method, resulting in 17 terms (Supplementary Figure S3); a manageable number for visualization.

We created a heatmap to examine the dynamics of these 17 terms over time (Figure 2, center right), which include cell cycle, processing of capped intron-containing pre-mRNA, DNA repair, apoptosis, and HIV infection. As shown in Supplementary Figure $\mathbf{S 3}$ c, we were able to expand 
resulting terms such as cell cycle to view aggregated and redundant terms removed in the compression process described above, such as nuclear envelope breakdown, DNA replication, and G2/M checkpoints, all of which are known bendamustine responses 23.

An enrichment analysis by itself provides no detail on how terms affect one another. Therefore, we constructed an annotated gene set network (AGN) for each time point (Figure 2, bottom). Each node in the AGN represents an enriched biological process, and the edges between the nodes reflect the directed connectivity between species that are annotated with that node's biological process term. As shown in Figure 2 and Supplementary File S5, this network can be visualized over time, where the node size will be adjusted based on the enriched value of the term at each time point, providing a high-level representation of the dynamics of signal flow, with animation if desired. These figures give insights into the high-level organization of signaling dynamics. At 30 seconds, the ontology term with the most changes involves processing of pre-mRNA, suggesting alterations in transcriptional activity. By 6 hours, terms involving genes associated with apoptosis, protein translation, and HIV response (explored in the next section) have their relative peaks at 6 hours. However, by the 12 and 24 hour time points, cell cycle becomes the dominant ontology term. This suggests early response is mediated through phosphorylation and changes in transcription, while later activity manifests through signaling activity. Looking at network connectivity, RNA polymerase II transcription is regulated by four terms (cellular response to stress, cell cycle, sumo E3 ligases sumoylate target proteins, HIV infection), but only regulates a single term (rho GTPase effectors), suggesting the transcription is highly regulated in response to bendamustine. The cell cycle node is connected to all other nodes in the AGN, in agreement with its importance 
in regulating response to bendamustine ${ }^{23}$. Overall, the AGN provides a broad representation of the data that allows users to visualize major cellular processes and their mutual interactions. Users can then identify terms of importance to be used as a starting point for further exploration.

\section{MAGINE elucidates DNA repair, cell cycle, and cell death proteins central to bendamustine}

response. We next examined how our data recapitulate existing knowledge of bendamustine's mechanism of action (the "canonical mechanism"). We extracted a molecular network from the AGN focused on the terms most commonly associated with bendamustine response ${ }^{\sqrt{23}}$ : DNA repair, cell cycle, and apoptosis (Figure 3, top center). There are a total of 65, 146, and 28 species in the network for the terms DNA repair, cell cycle, and apoptosis respectively. 33 species were classified as both DNA repair and cell cycle, 37 genes were classified as cell cycle and apoptosis, two genes were in all three terms, while no genes were classified as both DNA repair and apoptosis. We saw higher connectivity between DNA repair and cell cycle (132 outgoing and 117 incoming edges) and cell cycle and apoptosis (154 outgoing and 609 incoming edges) compared to DNA repair and apoptosis (24 outgoing and 56 incoming edges).

We next examined each of these ontology terms at the molecular level using MAGINE's network module. In the DNA repair term, we saw 173 phosphorylation events across 80 proteins, 43 changes in protein expression, and 5 changes of RNA expression (Figure 3, left). Of these, we identified proteins involved in DNA ligase (LIG1, LIG2), required to alleviate interstrand crosslinks, and double stranded break response MRN complex (RAD50, MRE11A, NBN) ${ }^{43}$, suggesting that the interstrand cross-links are corrected during DNA replication creation of double stranded 
breaks. We also identified proteins involved with base excision repair pathways (DDB1, XRCC1, XRCC2, XRCC5, XCCC6) and nucleotide excision repair (ERCC3, ERCC5, ERRC6) 44 , suggesting that the pyrimidine analog properties of bendamustine also contribute to DNA damage in addition to interstrand cross-links.

We next extracted and visualized changes in cell cycle status (Figure 3 , center). For the "cell cycle" term, we saw 444 significant phosphorylation events across 153 proteins, 111 changes in protein expression, and 17 changes of RNA expression. We clustered protein expression changes and created a sub-network of up-regulated species. We saw up-regulation of CDK1, AURKA, AURKB, CCNB1, CDC20, and PLK1 45,47. Of these, CDC20, CDK1, and CCNB1 regulate chromosome separation 48 , while expression of AURKA/AURKB/PLK1 regulates progress through the G2-M checkpoint ${ }^{46}$. Without this checkpoint, activated apoptotic signals could lead to mitotic catastrophe during metaphase/anaphase transition, a mechanism known to occur in response to bendamustine 23 .

Finally, we used the Subgraph module to explore downstream targets of CASP3, an effector caspase whose cleavage marks commitment to apoptosis 49 . Although significant changes in CASP3 concentration were not detected from the experimental datasets, enough biological evidence about CASP3 activation exists that motivate exploration of its downstream targets. To accomplish this, We created a network of downstream targets of CASP3 and visualized their expression levels over time (Figure 3, right). We saw 9 proteins that were down-regulated and one up-regulated at late time points, after apoptosis was induced. Thus, despite not measuring changes 
in CASP3, MAGINE allowed us to gather evidence suggesting its activation in a signaling capacity.

\section{Bendamustine regulation of nuclear pore proteins and regulators of nuclear trafficking: po-}

tential clinical significance to HIV infection. Throughout the exploration of the HL-60 response to bendamustine dataset, we noticed that the term HIV infection was a significantly enriched ontology term that apparently played no role in the cell-death response associated with bendamustine. However, since HIV infection hijacks and modulates cell cycle and DNA repair 50|51, we examined whether a plausible explanation of the enrichment in proteins associated with HIV infection is the overlap of underlying species with these expected biological process terms. The data showed that this overlap did not completely account for the finding (Figure 4). Proteins downregulated in response to bendamustine marked with the HIV infection term include those related to the nuclear pore (NUP107, NUP133, NUP153, NUP155, NUP160, NUP188, NUP210, NUP214, NUP35, NUP37, NUP50, NUP62, NUP88, NUP93, NUP98) as well as regulators of the trafficking through the nuclear pore (RANBP1, RANBP2). Perturbations to RANBP2 are known to disrupt the HIV virus' ability to shuttle the HIV-1 Rev protein between the cytoplasm and nucleus, making it a potential target for inhibition of HIV ${ }^{52}$. Additionally, CCNT1, a co-factor of the HIV-1 Tat protein necessary for full activation of viral transcription, is down-regulated at the 60 and 72 hour time points. Thus, the ability of bendamustine to perturb HIV infection-related genes could have clinical relevance. While treatment with bendamustine (for other disease indications) in HIVpositive patients is rare, case reports state that two HIV-positive patients with chronic lymphocytic leukemia received bendamustine without adverse outcomes 53 . Therefore, unbiased analysis of this 
dataset enabled side effects exploration that could lead to further leads in HIV treatment. Although such results clearly require further validation, they demonstrates the ability of MAGINE to identify non-canonical cellular pathways effected by drug treatment.

\section{DISCUSSION}

Experimental advances in -omics data generation have provided a wealth of data and opportunities to advance currently available tools. The most common analysis types include network and enrichment analysis, which were originally designed for single sample, single platform studies. These analyses are complementary: network analyses give molecular interaction insights, while enrichment analyses show the coordination of molecular activity at the level of biological processes. Although both approaches have been widely used, computational tools allowing users to employ both analyses in a complementary fashion on multi-sample, multi-omics data have been lacking. Here we demonstrated that MAGINE can integrate multi-sample -omic data with enrichment analysis and data-specific signaling networks in a single computational environment, which allows users to gain many types of biological insights and to explore their data interactively and efficiently.

MAGINE enables users to construct custom analysis protocols in Jupyter notebooks that are both reproducible and transferable. Existing software such as Biojupies ${ }^{54}$ and PaDua ${ }^{55}$ have demonstrated the power of this approach on RNAseq and phospho-proteomics analysis. We expect the use of Jupyter notebooks to increase and see MAGINE as highly complementary to such 
pipelines.

Multi-omics mesaurements provide larger cellular coverage than traditional in vitro/in cell measurements (Western blots or ELISA) and identification of unexpected significantly changed biochemical species is common. MAGINE enables users to elucidate these findings through exploration of the molecular data and their biological network context, which is often a slow step. Here, we demonstrated MAGINE's ability to quickly explore why the term HIV infection was detected and its relationship to known bendamustine responses. We expect this utility to be useful in identifying and exploring non-canonical cellular pathways that are often disregarded or ignored.

Our results show the complexity in interpreting multi-omics data, as the response to bendamustine involves multiple genes and cellular pathways. Single time point measurements do not capture an entire mechanism, with some events occurring early (phosphorylation events of DNA repair proteins), and some later (up-regulation of cell cycle proteins, cleavage events of apoptotic CASP3). Interactive exploration of these data enables users to piece together the mechanism and design further experiments for validation and follow-up exploration.

In summary, MAGINE enables users to easily switch between enrichment analysis, networks, and experimental data within an iterative, interactive framework. This allows users to vary resolutions (molecular/biological process, static/dynamic) depending on the question at hand, and generate new hypotheses. As improvements and falling costs with -omic data generation allow multiple sample acquisitions per perturbation, we envision the need for integrative tools such as MAGINE increasing significantly. 


\section{METHODS}

MAGINE software implementation MAGINE is implemented as a Python package. It leverages several existing Python packages, including pandas ${ }^{25}$, NumPy ${ }^{56}$, Bioservices ${ }^{57}$, Matplotlib ${ }^{38}$, Seaborn ${ }^{58}$, matplotlib-venn, and networkx ${ }^{41}$ packages. Optional but recommended dependencies include jupyter-notebook ${ }^{59}$, python-igraph ${ }^{39}$, py2 $^{5 y t o s c a p e}{ }^{37}$, and plotly ${ }^{60}$ Python packages and graphviz ${ }^{40}$ and Cytoscape ${ }^{111}$ software for additional visualization features.

Data format MAGINE is designed for quantitative datasets. These data are loaded from a single file in a tabular format, with columns identifying the sample, time point, and experimental platform. Due to the large number of possible experimental platforms, we generalized naming of columns to support a wide variety of data types. MAGINE requires the following column labels: identifier, label, species_type, significant, fold_change, p_value, source, and sample_id, with a sample table shown in Supplementary Table $\mathbf{S 1}$. This format uses a stacked data file notation (denormalized, in database parlance), which provides ease of editing and compatibility (a single, text format table is all that is needed) at the expense of data redundancy. For identifier, we use HGNC 61 and Human Metabolite Database (HMDB) ${ }^{35}$ IDs for genes and compounds, respectively (we also provide tools to map other identifiers to these formats). The label column can be used to store any other information, such as post-translational modifications, aliases, or molecular weights. The source and sample id are used to label the experimental platform and sample condition (i.e., time point). The species type column is used to state if the species is a metabolite or gene (we use "gene" to label all possible gene products and allow the source column to specify the type). The 


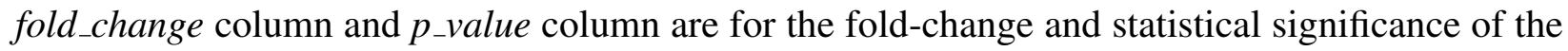
data compared to control (supplied from the raw data processing step performed before data are loaded into MAGINE). Note that the $p_{-}$value should be the adjusted value if using any form of multiple hypothesis testing. Finally, the significant column is used to identify if a given measurement is significant compared to control. The definition of significant is deliberately left to the user: in the situation where each experimental platform is handled by a different team, those teams can apply their own standards or software to defining significance, if desired.

Once loaded, data are stored in an instance of MAGINE's ExperimentalData class, built on the Pandas DataFrame with added methods designed for biological data. Our goal was to simplify and provide shorthand properties/methods to minimize the code needed to perform common operations such as filtering, querying, aggregating, and visualization of the data. A summary of these methods is shown in Supplementary Table $\mathbf{S 2}$ and example usage can be found online or in Supplementary Notebook S1]. MAGINE allows for custom workflows to be assembled from its component modules and methods (Figure 5). Python properties are constructed around the significant, source and sample_id columns, allowing users to gather only significant changes (data.sig), extract only label-free proteomics data (data.label_free), extract out only sample_id rows labeled "wild_type" (data.wild_type), or filter up-regulated fold-changed species (data.up). Importantly, filters can be chained together to create complex queries, with less complex syntax, and methods directly applied to the resulting information (data.label_free.sig.up.heatmap()). Identifiers or labels of these queries can then be aggregated (data.id_list or data.label_list) and passed to enrichment analysis or to construct a network. Additionally, the class has direct access to common biological 
plotting functions such as volcano plots (data.volcano()), heatmaps (data.heatmap ()$)$, or individual species plots (data.plot_species(...)).

Enrichment term aggregation MAGINE contains a method, remove_redundancy, for reducing the number of gene enrichment terms by aggregating redundant terms. First, it calculates the ratio of the sizes of the intersection and union (Jaccard index ${ }^{62}$ ) between genes within all term pairs. It then ranks all terms based on either their combined score (from EnrichR), number of genes in the term, or p-value. Starting from the highest ranked, it compares all lower ranked terms and removes them if their similarity is above a user-defined threshold, as shown in Supplementary Text 1 and demonstrated in Figure $\mathbf{S 3}$. This allows the user to minimize the number of total terms while maintaining a level of information content that preserves total information.

Annotated gene set network construction Annotated gene set networks start with a set of annotation terms, which can be selected based on expert knowledge, rank of enrichment, all compressed terms, or any other criteria. We first extract the set of nodes from a molecular network identified with the selected annotation terms. From there, we search through all possible combinations of pairs between the terms. For example, if term 1 has genes $(A, B, C)$ and term 2 has $(D, E)$, we count the number of edges from the possible sets ((A, D), (A, E), (B, D), (B, E), (C, D), (C, E)) that are found in the network edges. We then do the reverse (term 2 to term 1 ). If a node is in both sets, we consider the edges that connect the other term, not edges that are within the term. This is demonstrated in Supplementary Figure $\mathbf{S 5}$. 
Software availability and requirements MAGINE is released as open source software under the GNU General Public License, version 3. Source code for MAGINE can be found at github.com/LoLabVU/Magine, Full documentation can be found at magine.readthedocs.io. MAGINE has a full software test suite, which is executed using continuous integration and checked for code coverage. The project is version-controlled on GitHub. We encourage the community to post issues, questions, enhancement requests, and code contributions.

Experimental methods Briefly, HL-60 cells were plated and exposed to $100 \mathrm{M}$ bendamustine in triplicate for each time point, as described previously 4 . Sample preparation for transcriptomics, label-free proteomics, and metabolomics analyses was performed as previously described

4. SILAC and phSILAC samples were prepared in a similar manner as reported ${ }^{5}$, with the exception that SILAC samples were run by 1D chromatography rather than MudPIT. After introduction of drug, measurements were collected at 12 time points ranging from 30 seconds to 72 hours across 6 experimental platforms as shown in Figure S1 a. Each dataset was then formatted and combined into a single CSV to be used with MAGINE.

\section{ACKNOWLEDGEMENTS}

We thank Randi Gant-Branum, Lauren D. Palmer, Stacy D. Sherrod, D. Borden Lacy, Eric P. Skaar, and John A. McLean for data generation and feedback. We also thank Oscar Ortega, Lisa Poole, and Nate Braman for input and ideas, and Michael Ripperger for discussions regarding visualization tools. 
Research was sponsored by the U.S. Army Research Office and the Defense Advanced Research Projects Agency and was accomplished under Cooperative Agreement no. W911 NF-14-20022. The views and conclusions contained in this document are those of the authors and should not be interpreted as representing the official policies, either expressed or implied, of the Army Research Office, DARPA, or the U.S. Government. The U.S. Government is authorized to reproduce and distribute reprints for Government purposes notwithstanding any copyright notation herein. This work was supported in part by the National Science Foundation grant MCB-1411482 (C.F.L., J.C.P). This work was also supported in part by the National Institutes of Health cooperative agreement 1U01CA215845-01 (C.F.L.).

\subsubsection{Conflict of interest statement.}

None declared. 
1. Ruedi Aebersold and Matthias Mann. Mass spectrometry-based proteomics. Nature, 422:198207, 2003.

2. Timothy K Toby, Luca Fornelli, and Neil L Kelleher. Progress in top-down proteomics and the analysis of proteoforms. Annual review of analytical chemistry, 9:499-519, 2016.

3. Zhong Wang, Mark Gerstein, and Michael Snyder. Rna-seq: a revolutionary tool for transcriptomics. Nature reviews genetics, 10(1):57, 2009.

4. Danielle B. Gutierrez, Randi L. Gant-Branum, Carrie E. Romer, Melissa A. Farrow, Jamie L. Allen, Nikesh Dahal, Yuan-Wei Nei, Simona G. Codreanu, Ashley T. Jordan, Lauren D. Palmer, Stacy D. Sherrod, John A. McLean, Eric P. Skaar, Jeremy L. Norris, and Richard M. Caprioli. An integrated, high-throughput strategy for multiomic systems level analysis. Journal of Proteome Research, 17(10):3396-3408, 2018.

5. Jeremy L. Norris, Melissa A. Farrow, Danielle B. Gutierrez, Lauren D. Palmer, Nicole Muszynski, Stacy D. Sherrod, James C. Pino, Jamie L. Allen, Jeffrey M. Spraggins, Alex L.R. Lubbock, Ashley Jordan, William Burns, James C. Poland, Carrie Romer, M. Lisa Manier, Yuan Wei Nei, Boone M. Prentice, Kristie L. Rose, Salisha Hill, Raf Van De Plas, Tina Tsui, Nathaniel M. Braman, M. Ray Keller, Stacey A. Rutherford, Nichole Lobdell, Carlos F. Lopez, D. Borden Lacy, John A. McLean, John P. Wikswo, Eric P. Skaar, and Richard M. Caprioli. Integrated, high-throughput, multiomics platform enables data-driven construction of cellular responses and reveals global drug mechanisms of action. J. Proteome Res., 16:1364-1375, 2017. 
6. Lauren D. Palmer, Ashley T. Jordan, K. Nichole Maloney, Melissa A. Farrow, Danielle B. Gutierrez, Randi Gant-Branum, William J. Burns, Carrie E. Romer, Tina Tsui, Jamie L. Allen, William N. Beavers, Yuan-Wei Nei, Stacy D. Sherrod, D. Borden Lacy, Jeremy L. Norris, John A. McLean, Richard M. Caprioli, and Eric P. Skaar. Zinc intoxication induces ferroptosis in A549 human lung cells. Metallomics, 2019.

7. Aravind Subramanian, Pablo Tamayo, Vamsi K Mootha, Sayan Mukherjee, Benjamin L Ebert, Michael A Gillette, Amanda Paulovich, Scott L Pomeroy, Todd R Golub, Eric S Lander, et al. Gene set enrichment analysis: a knowledge-based approach for interpreting genome-wide expression profiles. Proceedings of the National Academy of Sciences, 102(43):15545-15550, 2005 .

8. Oliver D King, Rebecca E Foulger, Selina S Dwight, James V White, and Frederick P Roth. Predicting gene function from patterns of annotation. Genome research, 13(5):896-904, 2003.

9. Edward Y. Chen, Christopher M. Tan, Yan Kou, Qiaonan Duan, Zichen Wang, Gabriela V. Meirelles, Neil R. Clark, and Avi Ma'ayan. Enrichr: interactive and collaborative HTML5 gene list enrichment analysis tool. BMC Bioinformatics, 14, 2013.

10. Jing Wang, Suhas Vasaikar, Zhiao Shi, Michael Greer, and Bing Zhang. WebGestalt 2017: A more comprehensive, powerful, flexible and interactive gene set enrichment analysis toolkit. Nucleic Acids Research, 45(W1):W130-W137, 2017.

11. Paul Shannon, Andrew Markiel, Owen Ozier, Nitin S Baliga, Jonathan T Wang, Daniel Ramage, Nada Amin, Benno Schwikowski, and Trey Ideker. Cytoscape: a software environment 
for integrated models of biomolecular interaction networks. Genome research, 13(11):24982504, 2003.

12. Andreas Krämer, Jeff Green, Jack Pollard, and Stuart Tugendreich. Causal analysis approaches in ingenuity pathway analysis. Bioinformatics, 30:523-530, 2014.

13. Haiming Tang, Paul D. Thomas, Diane Kang, Caitlin Mills, Anushya Muruganujan, Xiaosong Huang, and Huaiyu Mi. PANTHER version 11: expanded annotation data from Gene Ontology and Reactome pathways, and data analysis tool enhancements. Nucleic Acids Research, 45(D1):D183-D189, 2016.

14. Da Wei Huang, Brad T. Sherman, Qina Tan, Joseph Kir, David Liu, David Bryant, Yongjian Guo, Robert Stephens, Michael W. Baseler, H. Clifford Lane, and Richard A. Lempicki. DAVID Bioinformatics Resources: expanded annotation database and novel algorithms to better extract biology from large gene lists. Nucleic Acids Res., 35:169-175, 2007.

15. Kakajan Komurov, Serkan Dursun, Serkan Erdin, and Prahlad T. Ram. NetWalker: A contextual network analysis tool for functional genomics. BMC Genomics, 13:282, 2012.

16. Fredrik Wrede and Andreas Hellander. Smart computational exploration of stochastic gene regulatory network models using human-in-the-loop semi-supervised learning. Bioinformatics, 35(24):5199-5206, 2019.

17. Jason A Papin, Joerg Stelling, Nathan D Price, Steffen Klamt, Stefan Schuster, and Bernhard O Palsson. Comparison of network-based pathway analysis methods. Trends in biotechnology, 22(8):400-405, 2004. 
18. Da Wei Huang, Brad T Sherman, and Richard A Lempicki. Bioinformatics enrichment tools: paths toward the comprehensive functional analysis of large gene lists. Nucleic acids research, 37(1):1-13, 2008.

19. Vikram Narayan, Tony Ly, Ehsan Pourkarimi, Alejandro Brenes Murillo, Anton Gartner, Angus I. Lamond, and Cynthia Kenyon. Deep Proteome Analysis Identifies Age-Related Processes in C. elegans. Cell systems, 3(2):1-16, 2016.

20. Francesca Sacco, Alessandra Silvestri, Daniela Posca, Stefano Pirrò, Pier Federico Gherardini, Luisa Castagnoli, Matthias Mann, and Gianni Cesareni. Deep proteomics of breast cancer cells reveals that metformin rewires signaling networks away from a pro-growth state. Cell Syst., 2:159-171, 2016.

21. Ruedi Aebersold and Matthias Mann. Mass-spectrometric exploration of proteome structure and function. Nature, 537:347-355, 2016.

22. Donald E. Knuth. Literate Programming. Computers and Chemical Engineering, 22(12):1745-1747, 1984.

23. Lorenzo M. Leoni and John A. Hartley. Mechanism of Action: The Unique Pattern of Bendamustine-Induced Cytotoxicity. Seminars in Hematology, 48:S12-S23, 2011.

24. Bruce D. Cheson and Mathias J. Rummel. Bendamustine: Rebirth of an old drug. Journal of Clinical Oncology, 27(9):1492-1501, 2009.

25. Wes McKinney et al. Data structures for statistical computing in python, 2010. 
26. Da Wei Huang, Brad T. Sherman, and Richard A. Lempicki. Bioinformatics enrichment tools: Paths toward the comprehensive functional analysis of large gene lists. Nucleic Acids Res., 37(1):1-13, 2009.

27. Maxim V. Kuleshov, Matthew R. Jones, Andrew D. Rouillard, Nicolas F. Fernandez, Qiaonan Duan, Zichen Wang, Simon Koplev, Sherry L. Jenkins, Kathleen M. Jagodnik, Alexander Lachmann, Michael G. McDermott, Caroline D. Monteiro, Gregory W. Gundersen, and Avi Ma'ayan. Enrichr: a comprehensive gene set enrichment analysis web server 2016 update. Nucleic Acids Res., 44:W90-W97, 2016.

28. Hiroyuki Ogata, Susumu Goto, Kazushige Sato, Wataru Fujibuchi, Hidemasa Bono, and Minoru Kanehisa. KEGG: Kyoto encyclopedia of genes and genomes. Nucleic Acids Res., 27:29-34, 1999.

29. Minoru Kanehisa, Yoko Sato, Masayuki Kawashima, Miho Furumichi, and Mao Tanabe. KEGG as a reference resource for gene and protein annotation. Nucleic Acids Res., 44:D457D462, 2016.

30. Livia Perfetto, Leonardo Briganti, Alberto Calderone, Andrea Cerquone Perpetuini, Marta Iannuccelli, Francesca Langone, Luana Licata, Milica Marinkovic, Anna Mattioni, Theodora Pavlidou, Daniele Peluso, Lucia Lisa Petrilli, Stefano Pirró, Daniela Posca, Elena Santonico, Alessandra Silvestri, Filomena Spada, Luisa Castagnoli, and Gianni Cesareni. SIGNOR: a database of causal relationships between biological entities. Nucleic Acids Res., 44:D548D554, 2016. 
31. Guanming Wu, Xin Feng, and Lincoln Stein. A human functional protein interaction network and its application to cancer data analysis. Genome Biol., 11:R53, 2010.

32. Andrew Chatr-Aryamontri, Rose Oughtred, Lorrie Boucher, Jennifer Rust, Christie Chang, Nadine K. Kolas, Lara O’Donnell, Sara Oster, Chandra Theesfeld, Adnane Sellam, Chris Stark, Bobby Joe Breitkreutz, Kara Dolinski, and Mike Tyers. The BioGRID interaction database: 2017 update. Nucleic Acids Res., 45:D369-D379, 2017.

33. C. Stark. BioGRID: a general repository for interaction datasets. Nucleic Acids Res., 34:D535D539, 2006.

34. David S. Wishart, Craig Knox, An Chi Guo, Roman Eisner, Nelson Young, Bijaya Gautam, David D. Hau, Nick Psychogios, Edison Dong, Souhaila Bouatra, Rupasri Mandal, Igor Sinelnikov, Jianguo Xia, Leslie Jia, Joseph A. Cruz, Emilia Lim, Constance A. Sobsey, Savita Shrivastava, Paul Huang, Philip Liu, Lydia Fang, Jun Peng, Ryan Fradette, Dean Cheng, Dan Tzur, Melisa Clements, Avalyn Lewis, Andrea De souza, Azaret Zuniga, Margot Dawe, Yeping Xiong, Derrick Clive, Russ Greiner, Alsu Nazyrova, Rustem Shaykhutdinov, Liang Li, Hans J. Vogel, and Ian Forsythei. HMDB: a knowledgebase for the human metabolome. Nucleic Acids Res., 37:603-610, 2009.

35. David S. Wishart, Dan Tzur, Craig Knox, Roman Eisner, An Chi Guo, Nelson Young, Dean Cheng, Kevin Jewell, David Arndt, Summit Sawhney, Chris Fung, Lisa Nikolai, Mike Lewis, Marie Aude Coutouly, Ian Forsythe, Peter Tang, Savita Shrivastava, Kevin Jeroncic, Paul Stothard, Godwin Amegbey, David Block, David D. Hau, James Wagner, Jessica Miniaci, 
Melisa Clements, Mulu Gebremedhin, Natalie Guo, Ying Zhang, Gavin E. Duggan, Glen D. MacInnis, Alim M. Weljie, Reza Dowlatabadi, Fiona Bamforth, Derrick Clive, Russ Greiner, Liang Li, Tom Marrie, Brian D. Sykes, Hans J. Vogel, and Lori Querengesser. HMDB: the Human Metabolome Database. Nucleic Acids Res., 35:521-526, 2007.

36. Max Franz, Christian T Lopes, Gerardo Huck, Yue Dong, Onur Sumer, and Gary D Bader. Cytoscape. js: a graph theory library for visualisation and analysis. Bioinformatics, 32(2):309_ $311,2015$.

37. Keiichiro Ono, Tanja Muetze, Georgi Kolishovski, Paul Shannon, and Barry Demchak. Cyrest: Turbocharging cytoscape access for external tools via a restful api. F1000Research, 4, 2015.

38. John D Hunter. Matplotlib: A 2d graphics environment. Computing in science \& engineering, 9(3):90, 2007.

39. Gabor Csardi, Tamas Nepusz, et al. The igraph software package for complex network research. InterJournal, Complex Systems, 1695(5):1-9, 2006.

40. John Ellson, Emden Gansner, Lefteris Koutsofios, Stephen C North, and Gordon Woodhull. Graphvizopen source graph drawing tools. In International Symposium on Graph Drawing, pages 483-484. Springer, 2001.

41. A Hagberg, D Schult, and P Swart. Networkx: Python software for the analysis of networks. Mathematical Modeling and Analysis, Los Alamos National Laboratory, 2005.

42. Paul Harris and Peter Ralph. Human leukemic models of myelomonocytic development: a review of the hl-60 and u937 cell lines. Journal of leukocyte biology, 37(4):407-422, 1985. 
43. Jaco H Houtgraaf, Jorie Versmissen, and Wim J van der Giessen. A concise review of dna damage checkpoints and repair in mammalian cells. Cardiovascular Revascularization Medicine, 7(3):165-172, 2006.

44. Jan HJ Hoeijmakers. Nucleotide excision repair ii: from yeast to mammals. Trends in Genetics, 9(6):211-217, 1993.

45. Jiri Bartek and Jiri Lukas. Chk1 and chk2 kinases in checkpoint control and cancer. Cancer cell, 3(5):421-429, 2003.

46. Anna S Nikonova, Igor Astsaturov, Ilya G Serebriiskii, Roland L Dunbrack, and Erica A Golemis. Aurora a kinase (aurka) in normal and pathological cell division. Cellular and Molecular Life Sciences, 70(4):661-687, 2013.

47. Katrien Vermeulen, Dirk R Van Bockstaele, and Zwi N Berneman. The cell cycle: a review of regulation, deregulation and therapeutic targets in cancer. Cell proliferation, 36(3):131-149, 2003.

48. Alessandro Torgovnick and Björn Schumacher. Dna repair mechanisms in cancer development and therapy. Frontiers in genetics, 6:157, 2015.

49. Elizabeth A Slee, Mary T Harte, Ruth M Kluck, Beni B Wolf, Carlos A Casiano, Donald D Newmeyer, Hong-Gang Wang, John C Reed, Donald W Nicholson, Emad S Alnemri, et al. Ordering the cytochrome c-initiated caspase cascade: hierarchical activation of caspases-2,3,-6,-7,-8, and-10 in a caspase-9-dependent manner. The Journal of cell biology, 144(2):281292, 1999. 
50. Mira S Chaurushiya and Matthew D Weitzman. Viral manipulation of dna repair and cell cycle checkpoints. DNA repair, 8(9):1166-1176, 2009.

51. Eric H Humphries and Howard M Temin. Cell cycle-dependent activation of rous sarcoma virus-infected stationary chicken cells: avian leukosis virus group-specific antigens and ribonucleic acid. Journal of virology, 10(1):82-87, 1972.

52. Ruonan Zhang, Rajeev Mehla, and Ashok Chauhan. Perturbation of host nuclear membrane component RanBP2 impairs the nuclear import of human immunodeficiency virus -1 preintegration complex (DNA). PLoS ONE, 5(12), 2010.

53. Naoki Shimada, Kaoru Uchimaru, Koichiro Yuji, Naoki Oyaizu, Arinobu Tojo, Tomohiko Koibuchi, and Nobuhiro Ohno. Treatment of chronic lymphocytic leukemia with bendamustine in an HIV-infected patient on antiretroviral therapy: a case report and review of the literature. Clinical Case Reports, 3(6):453-460, 2015.

54. Denis Torre, Alexander Lachmann, and Avi Ma'ayan. BioJupies: Automated Generation of Interactive Notebooks for RNA-Seq Data Analysis in the Cloud. Cell Systems, 7(5):556561.e3, 2018.

55. Anna Ressa, Martin Fitzpatrick, Henk Van Den Toorn, Albert J.R. Heck, and Maarten Altelaar. PaDuA: A Python Library for High-Throughput (Phospho)proteomics Data Analysis. Journal of Proteome Research, 18(2):576-584, 2019.

56. Stefan Van Der Walt, S Chris Colbert, and Gael Varoquaux. The numpy array: a structure for efficient numerical computation. Computing in Science \& Engineering, 13(2):22, 2011. 
57. Thomas Cokelaer, Dennis Pultz, Lea M. Harder, Jordi Serra-Musach, Julio Saez-Rodriguez, and Alfonso Valencia. BioServices: A common Python package to access biological Web Services programmatically. Bioinformatics, 29(24):3241-3242, 2013.

58. Michael Waskom et al. mwaskom/seaborn: v0.8.1 (september 2017), September 2017.

59. Thomas Kluyver, Benjamin Ragan-Kelley, Fernando Pérez, Brian E Granger, Matthias Bussonnier, Jonathan Frederic, Kyle Kelley, Jessica B Hamrick, Jason Grout, Sylvain Corlay, et al. Jupyter notebooks-a publishing format for reproducible computational workflows. In ELPUB, pages 87-90, 2016.

60. Plotly Technologies Inc. Collaborative data science, 2015.

61. Elspeth A Bruford, Michael J Lush, Mathew W Wright, Tam P Sneddon, Sue Povey, and Ewan Birney. The hgnc database in 2008: a resource for the human genome. Nucleic acids research, 36(suppl_1):D445-D448, 2007.

62. G. Gilbert. Distance between sets. Nature, 239(5368):174, 1972. 
bioRxiv preprint doi: https://doi.org/10.1101/2020.03.02.974121; this version posted March 3, 2020. The copyright holder for this preprint (which was not certified by peer review) is the author/funder, who has granted bioRxiv a license to display the preprint in perpetuity. It is made available under aCC-BY-NC 4.0 International license.

\section{Figures}




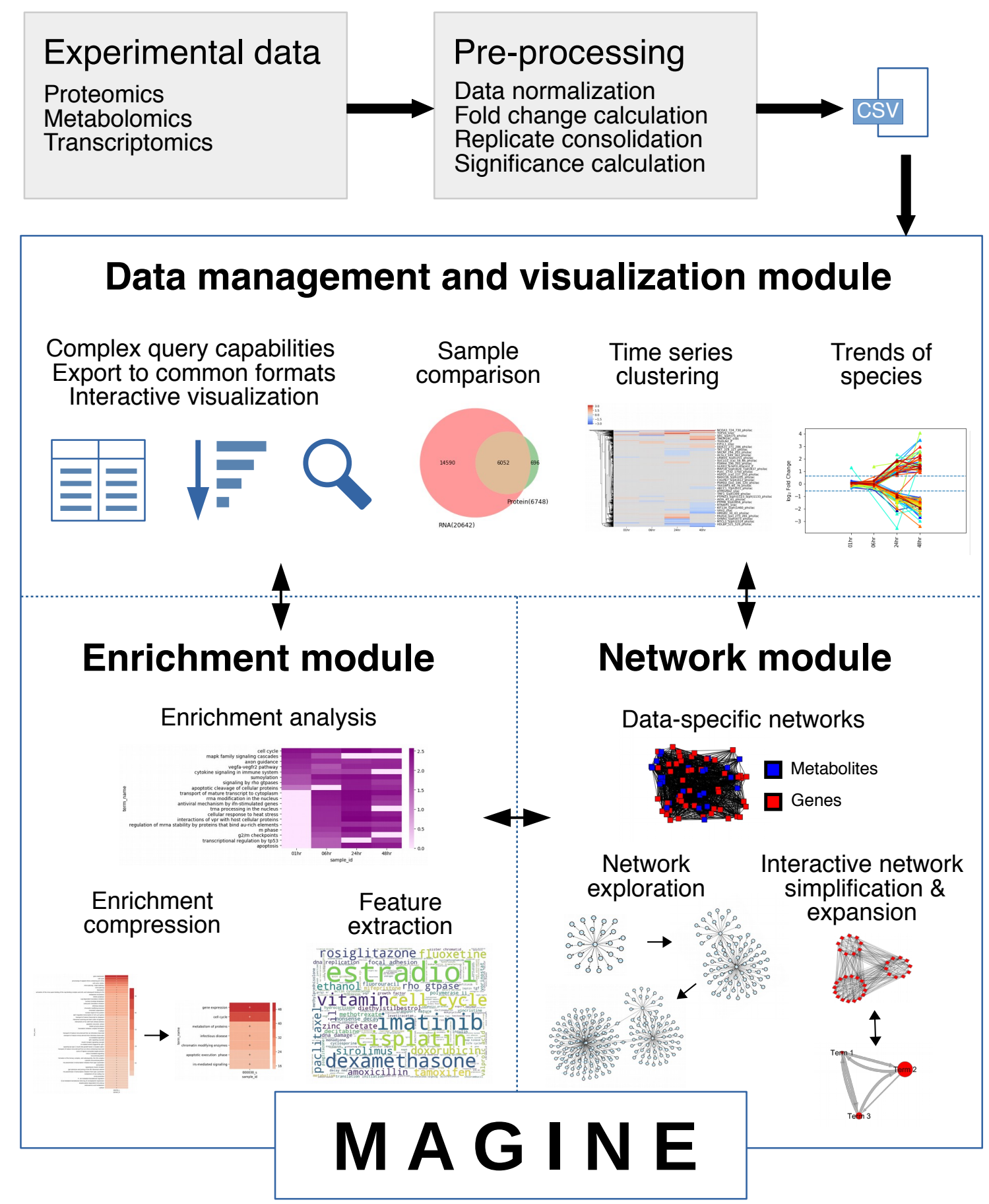

Figure 1: The MAGINE platform. MAGINE is designed for quantitative time-series multi-omic data. It is built around three concepts: data management, enrichment analysis, and network exploration. The modular design allows flow of information among the data, enrichment, and network modules, allowing an iterative cycle with varying levels of resolution. Example outputs of each module are provided in each panel. 
Multi-omics data

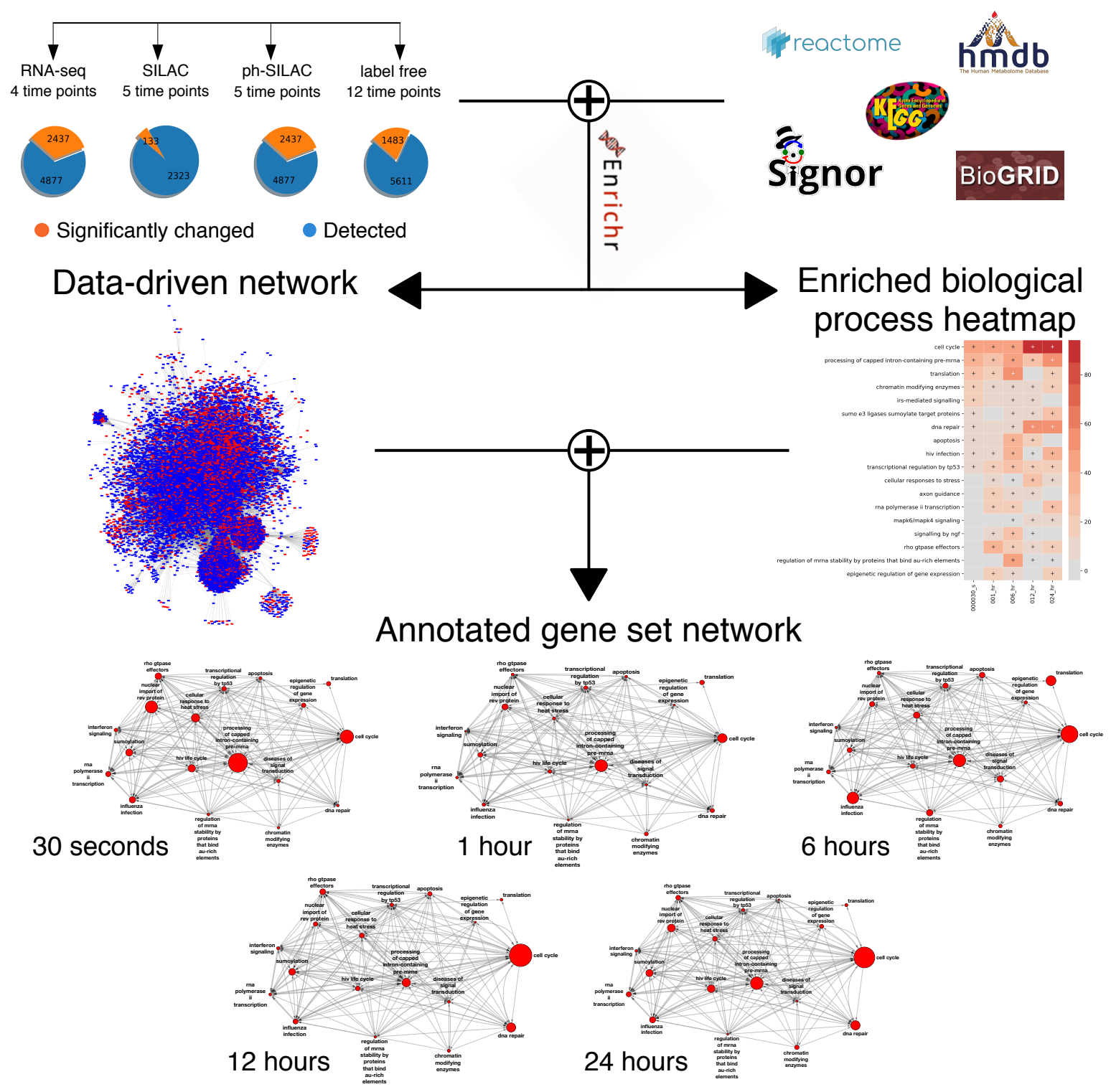

Figure 2: Example workflow of bendamustine mechanism extraction. Multi-omics datasets (top left) are combined with public databases (top right) to generate detailed interaction networks (middle left) and enriched annotated gene set (AGS) heatmaps (middle right). The data-derived network is then pruned by extracting a subnetwork of genes from high-ranking AGS terms and collapsed to produce annotation-level networks (bottom), where each node is labeled by an AGS term and scaled according to enrichment of that term; the width of each edge represents the number of edges between the terms. The network can be viewed at each time point or as an animation (Supplementary File S5). 


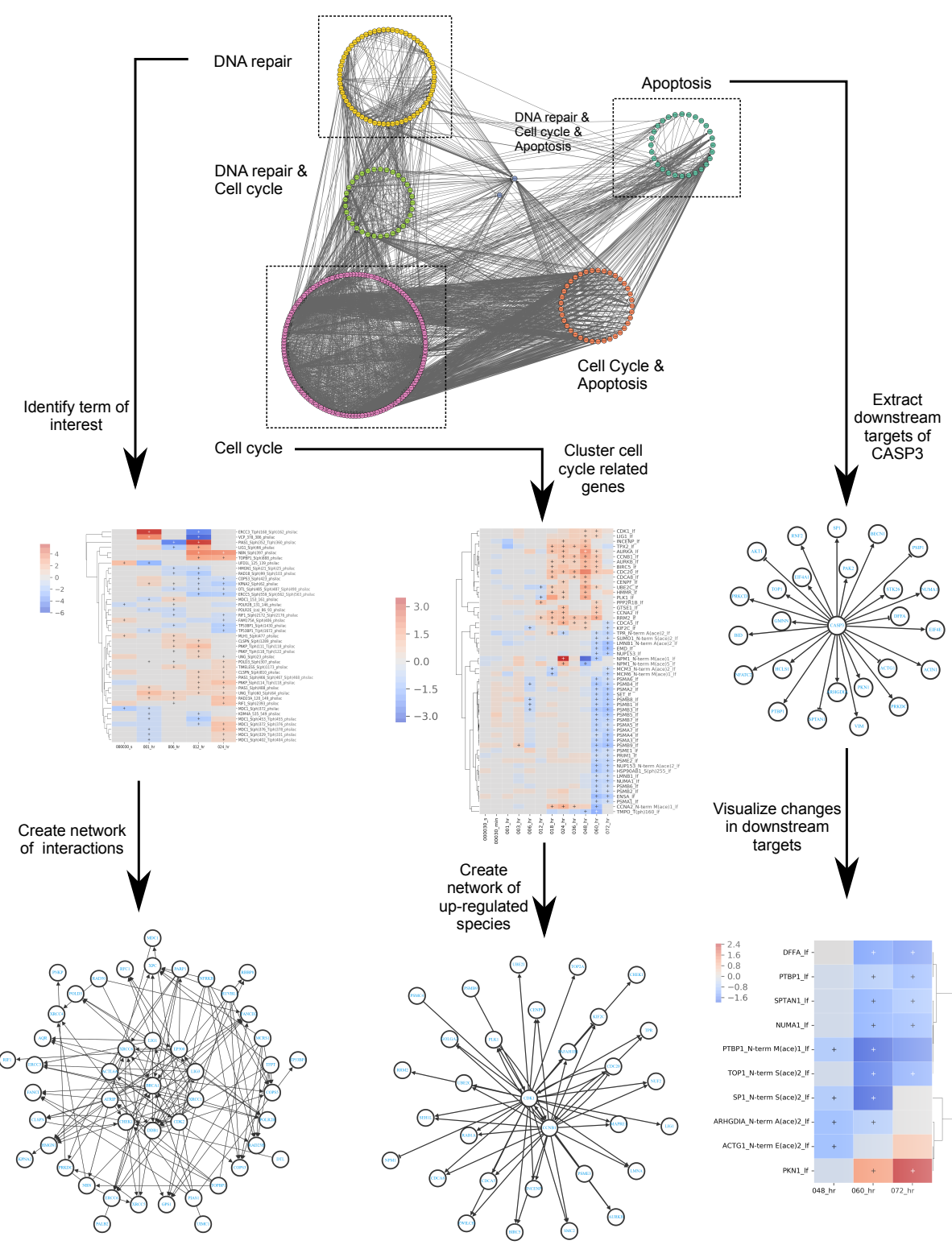

Figure 3: Custom workflows for exploring known biological processes of bendamustine. Molecular network constructed from DNA repair, cell cycle, and apoptosis classified species, the canonical processes involved in bendamustine response (top). The network is an expanded version of the annotated gene network from Figure 2, From the term "DNA repair", MAGINE can visualize (center left) as well as construct a signaling network corresponding to the term's molecular species (bottom left). Due to CASP3 importance in executing apoptosis, we constructed a network of downstream species (center right). We then filtered the experimental data to generate a heatmap (bottom right). Eight of nine species are down-regulated, indicating CASP3 activity. We then visualized the changes to cell cycle-related genes over time (center), and subsequently constructed a molecular network based on CCNB1 and CDK1 (middle bottom), two genes responsible for G2-M transition of cell cycle. 

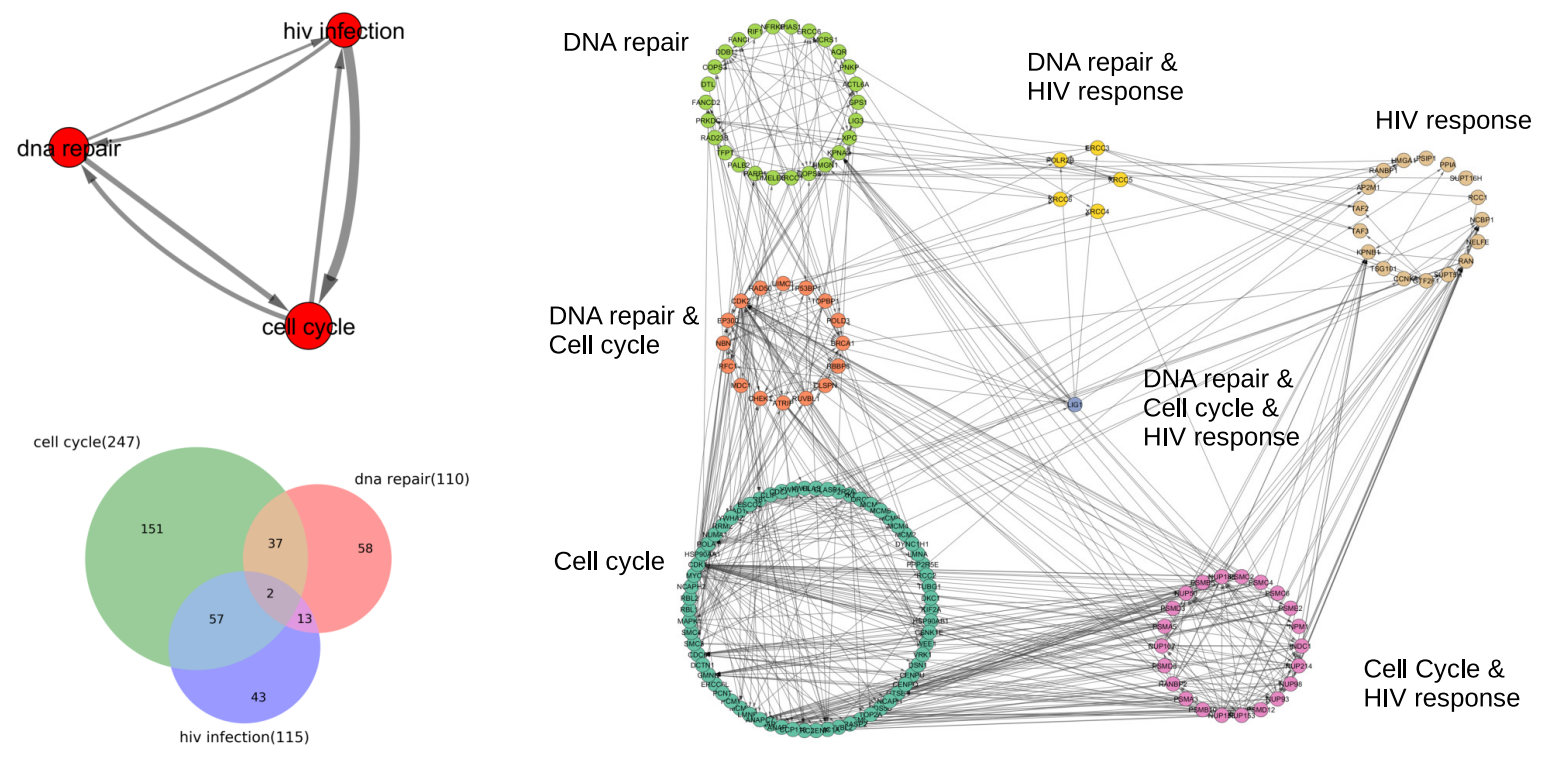

Figure 4: Common genes between bendamustine mechanism processes and the GO term $H I V$ infection. An unexpected outcome of the analysis revealed that proteins related to the GO term $H I V$ infection were modulated with bendamustine. An annotated gene set network of the interaction between the GO terms DNA repair, HIV infection, and cell cycle (top left). The overlap between genes labeled in each pathway labeled by Reactome (bottom left). Molecular interaction network of all significantly changed species grouped according to their classified pathway (right). 


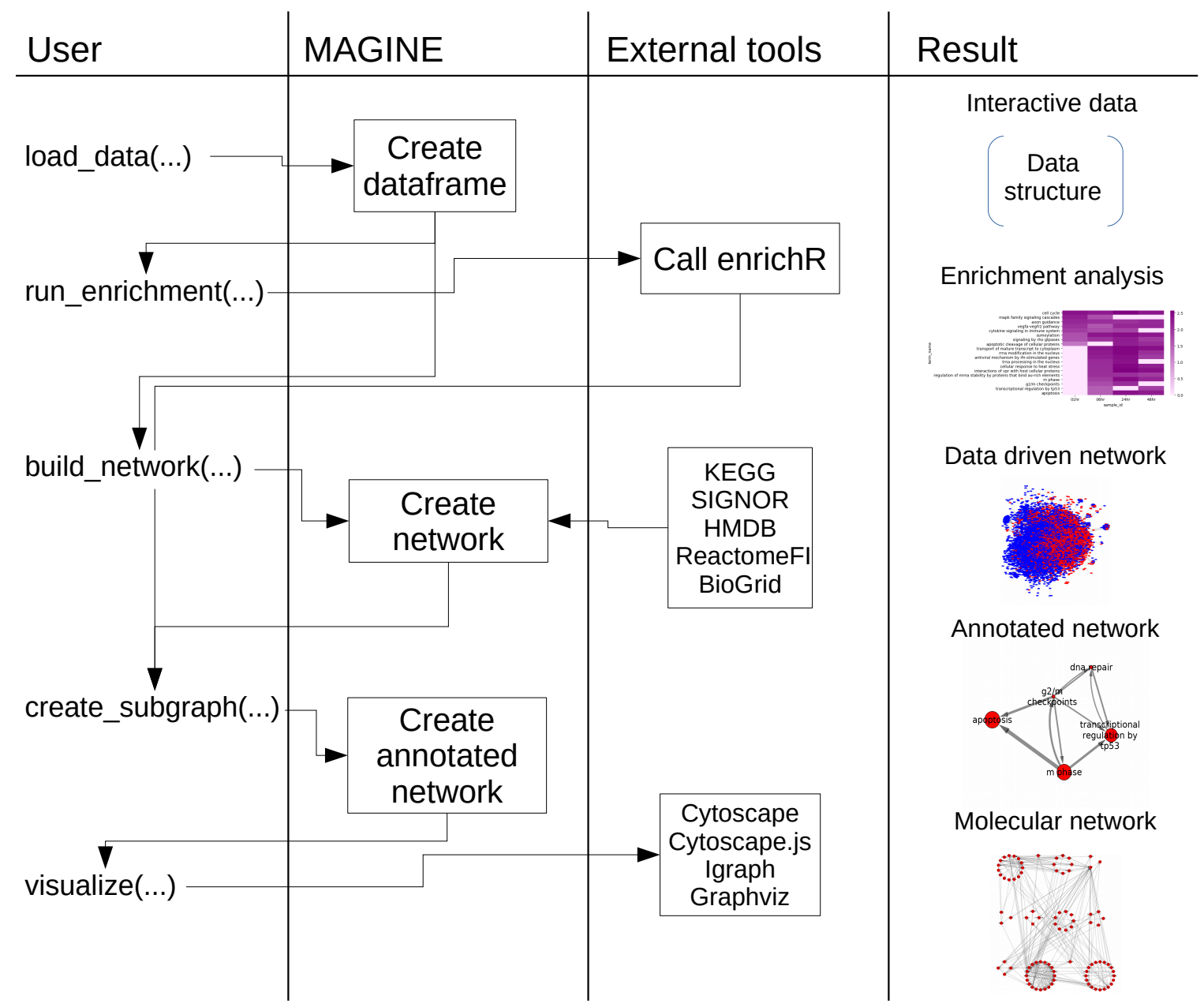

Figure 5: Example of MAGINE workflow. The figure shows example function calls made by the user (left column) and how these are handled by MAGINE and external tools (middle columns), and examples of resulting output (right column). This is a typical processing workflow where users desire to run enrichment analysis, construct a data-specific network, and construct a compressed annotated gene set network. 